\title{
Breathers in systems with intrinsic and extrinsic nonlinearities
}

\author{
L. Cruzeiro-Hansson ${ }^{1}$, J. C. Eilbeck, J. L. Marín, F. M. Russell \\ Department of Mathematics, Heriot-Watt University, Edinburgh EH14 4AS, U.K.
}

\begin{abstract}
We study the interplay between nonlinearity and localization in a model consisting of a quantum quasiparticle interacting with a nonlinear extended lattice. The isolated lattice can support discrete breathers, and the coupling of the quasiparticle to the lattice leads to localized quasiparticle states. Minimum energy states of the full system can have both a breather and a solitonic character. Excited states lead to interesting new phenomena, such as full breather states, which arise from the combination of intrinsic and extrinsic nonlinearity.
\end{abstract}

Key words: Polarons; Discrete Breathers PACS: $71.50+\mathrm{t}, 63.20 \mathrm{Kr}, 71.25 . \mathrm{Cx}$

\section{Introduction}

Experimental evidence for intrinsic localized modes (ILMs) has been accumulating recently [1-4]. For example, intrinsic localized spin waves have been generated with a microwave pulse [1], and localized vibrational modes have been detected by the structure and redshifts of the overtone Raman spectra of a halide-bridged mixed-valence transition metal complex [2]. The latter is formally very similar to the experiments performed in the early 80's by Careri and co-workers [5] (for a review see [6]), in which a spectral line, particularly visible at low temperature, was observed in a crystal of acetanilide. Here the shifts in the overtone spectrum could be accounted for, in a quantitative manner, by a nonlinear coupling of the amide I mode to optical phonons $[7,6]$. Finally, following a proposal by Floría et al. [8], localized excitations in Josephson junction ladders have been detected in I/V curves [3] and visualized by laser microscopy [4].

$\overline{1}$ Corresponding author. E-mail: leonor@ma.hw.ac.uk. 
ILMs in classical lattices are also known generically as discrete breathers (DBs). They were first recognized as a general phenomenon in the works of Takeno and Sievers [9], and the extent of this genericity was rigorously proven in $[10]$. For a review, see $[11,12]$. On the other hand, a different area, also concerned with nonlinear localization and with a longer history, is that of solitons. Strictly speaking, a soliton is a localized solution of a completely integrable system. By extension, many nonlinear localized solutions in non-integrable systems are also called solitons $[13,14]$. This loose terminology can be confusing, and in addition some solutions which have for many years been called solitons are perhaps better described as breathers. This is the case of some nonlinear-Schrödinger class of lattices, like the discrete self-trapping equation (DST) [15]. Conversely, the term "breather" was hijacked from an integrable system (the sine-Gordon equation). However, DBs are more generic than solitons in discrete systems, since they do not require complete integrability and arise from an interplay of nonlinearity and lattice discreteness [11]. As a working definition here we will designate as DBs those localized solutions with at least two degrees of freedom in a one dimensional lattice (i.e. they have an "internal" degree of freedom, a vibrational motion). Stable localized solutions with one degree of freedom we will call solitons.

The Davydov model for interaction between a quantum quasiparticle and a lattice exhibits solitons. In the original model $[6,16]$, one arrives at a continuumapproximation description which is essentially the nonlinear Schrödinger equation (NLS). In the discrete model, one also finds localized stationary solutions (which, by extension, we keep calling Davydov solitons). The Davydov model is very similar to the Holstein model for the interaction of an electron with a polarizable lattice $[17,18]$. In both, the origin for the nonlinearity lies only in the coupling between the lattice vibrations and the quasiparticle. In most studies, the lattice Hamiltonian and the quasiparticle Hamiltonian are both linear.

We wish to extend the previous studies to nonlinear lattices, where DBs are also possible. Hence, our Hamiltonian has two sources of nonlinearity: that inherent in the lattice, and an extrinsic one due to the quasiparticle-lattice interaction. There have been previous works which have analyzed the effect of introducing lattice nonlinearities in the polaron problem. Davydov[19] studied an extension to his model which included a nonlinear lattice, while Kenkre et al. [20-22] showed the non-trivial effects that lattice nonlinearities have in the context of the general polaron problem, under a semiclassical (DNLS) approximation (a recent study [23] extended these findings to a fully quantummechanical treatment). Gaididei et al. [24] have also studied a related problem. All these works were centered on the the ground state or stationary states of the polaron.

However in this paper we wish to take advantage of the lattice nonlinearity to 
be able to produce discrete breathers. We investigate DBs as excited states of the soliton/polaron states of such systems. This is done in the framework of a mixed quantum-classical, non-adiabatic treatment, which allows to compute the dynamical evolution of the system. This work can be seen as a preliminary step in the study of interactions between neutral DBs and polarons.

In the next section we introduce the Hamiltonian and describe the minimum energy solutions of this Hamiltonian. In Section 3 we describe the states that arise due to different excitations of the minimum energy states.

\section{The Hamiltonian and its minimum energy states - the solito- breathers.}

The Hamiltonian $\hat{H}$ has three parts:

$$
\hat{H}=\hat{H}_{\mathrm{qp}}+\hat{H}_{\mathrm{qp}-\mathrm{ph}}+H_{\mathrm{ph}}
$$

where $\hat{H}_{\mathrm{qp}}$ is the Hamiltonian for a quasiparticle, $\hat{H}_{\mathrm{qp}-\mathrm{ph}}$ describes the interaction of the quasiparticle with the lattice and $H_{\mathrm{ph}}$ is the lattice Hamiltonian.

The Hamiltonian for the quasiparticle has the usual tight binding form:

$$
\hat{H}_{\mathrm{qp}}=\epsilon \sum_{n=1}^{N} \hat{a}_{n}^{\dagger} \hat{a}_{n}-V \sum_{n=1}^{N}\left(\hat{a}_{n} \hat{a}_{n-1}^{\dagger}+\hat{a}_{n}^{\dagger} \hat{a}_{n+1}\right)
$$

where $\hat{a}_{n}^{\dagger}$ is the creation operator for a quasiparticle in site $n, \epsilon$ is the selfenergy of the quasiparticle and $V$ the transfer term for the quasiparticle to move between neighbouring sites.

The Hamiltonian for the interaction of the quasiparticle with the lattice includes the coupling to acoustic (or Debye) phonons:

$$
\hat{H}_{\mathrm{qp}-\mathrm{ph}}=\chi \sum_{n=1}^{N}\left[\left(u_{n+1}-u_{n-1}\right) \hat{a}_{n}^{\dagger} \hat{a}_{n}\right]
$$

where $\chi$ is a parameter which describes the strength of the quasiparticle-lattice interaction.

Hamiltonians of this form have been used in many contexts, from polarons to bioenergetics [6]. The main difference of the present work with respect to those studies is the form of the phonon Hamiltonian, which we take as follows: 


$$
\begin{aligned}
& H_{\mathrm{ph}}=H_{\mathrm{ph}}^{\mathrm{c}}+H_{\mathrm{ph}}^{\mathrm{o}} \\
& H_{\mathrm{ph}}^{\mathrm{c}}=\frac{\kappa a^{2}}{72} \sum_{n=1}^{N}\left[\left(\frac{a}{a+u_{n}-u_{n-1}}\right)^{12}-2\left(\frac{a}{a+u_{n}-u_{n-1}}\right)^{6}\right] \\
& H_{\mathrm{ph}}^{\mathrm{o}}=\kappa^{\prime} \sum_{n=1}^{N}\left(\frac{1}{2} u_{n}^{2}+\frac{1}{4} u_{n}^{4}\right)+\frac{1}{2 M} \sum_{n=1}^{N} p_{n}^{2} .
\end{aligned}
$$

Here $u_{n}$ is the displacement from equilibrium position of site $n, p_{n}$ is the momentum of site $n, a$ is the equilibrium distance between sites, $\kappa$ is the elasticity constant of the nonlinear lattice and $\kappa^{\prime}$ is a similar constant for the on-site potential. The coupling interactions between sites are described by a Lennard-Jones potential $H_{\mathrm{ph}}^{\mathrm{c}}$, a potential commonly used to describe interactions between atoms. Finally, the on-site potential $H_{\mathrm{ph}}^{\mathrm{o}}$ is as used in many breather studies [11]. It can be considered to represent the effect of the rest of the crystal (in a mean field approach) on the one dimensional chain whose states are studied explicitly.

Since we will be dealing with localized solutions, it is very useful to know the spectrum of linear excitations for the lattice in its ground state. This is the classical "phonon band" of the lattice, and it gives a good approximation to the spectrum for small perturbations which are not localized around the polaron or breather. For our lattice Hamiltonian (4), the phonon dispersion relation reads:

$$
\omega_{\mathrm{ph}}^{2}=\frac{\kappa^{\prime}}{M}+4 \frac{\kappa}{M} \sin ^{2}(q / 2), \quad-\frac{\pi}{2} \leq q \leq \frac{\pi}{2}
$$

We adopt a mixed quantum-classical approach in which the lattice is treated classically, while the quasiparticle is treated quantum mechanically. Accordingly, the displacements $u_{n}$ and momenta $p_{n}$ are real variables. The quasiparticle variables are operators, a distinction which is marked by the hats above the corresponding variables. This is a natural extension of classical breather studies [11]. It is a mixed quantum-classical approach which neglects the quantum uncertainty in the lattice positions and momenta [25]. It is particularly applicable when the mass of the sites is large. Its validity was examined as a function of temperature (in the context of the Davydov model) in a previous study [26]. The main difference between the full quantum approach and the mixed quantum-classical approach is that the former treats the lattice motion quantum-mechanically, while the latter treats it classically. Thus the lattice distortion, correlated with the position of the quantum quasiparticle, was chosen as a particularly relevant quantity to compare. The simulations showed that, at $2.8 \mathrm{~K}$, the mixed quantum-classical model predicts a lattice distortion that is $6 \%$ smaller than that predicted by the full quantum model [26]. This difference decreases rapidly as the temperature increases, and is negligible above $11 \mathrm{~K}$. When the aim is to make quantitative comparisons 
with experimental data, and depending on the precision of the experimental measurements, it may be necessary to treat the lattice quantum mechanically. But the aim here is to gain a qualitative insight into the type of solutions that can arise in these systems, rather than achieving quantitative precision. The mixed classical-quantum treatment has a much lower computational complexity, and allows one to study exact solutions in large-sized systems and with great detail [25]. It should also be noted that it is different from the adiabatic approximation which corresponds to assuming that the mass of the sites is much larger than that of the quantum quasiparticle. Indeed, while the adiabatic approximation is valid when the lattice is infinitely slower than the quasiparticle, the mixed quantum-classical approach makes no assumption about the time-scales of these two degrees of freedom, as is apparent from the equations of motion below.

The mixed quantum-classical regime is ideal as the behaviour of the system can be probed without any approximations. Indeed, one of its main advantages is that it is trivial to show that the exact wavefunction for Hamiltonian (1-4) is:

$$
\left|\psi(t)>=\sum_{n=1}^{N} \varphi_{n}\left(\left\{u_{n}\right\},\left\{p_{n}\right\}, t\right) \hat{a}_{n}^{\dagger}\right| 0>
$$

where $\varphi_{n}$ is the probability amplitude for a quasiparticle in site $n$ and the wavefunction is a superposition of states for the quasiparticle to be in each site. The probability amplitude is dependent on the lattice displacements and momenta in a way that is specified only by the equations of motion. These are:

$$
\begin{aligned}
i \hbar \frac{d \varphi_{n}}{d t} & =\epsilon \varphi_{n}-V\left(\varphi_{n+1}+\varphi_{n-1}\right)+\chi\left(u_{n+1}-u_{n-1}\right) \varphi_{n} \\
M \frac{d^{2} u_{n}}{d t^{2}} & =-\chi\left(\left|\varphi_{n-1}\right|^{2}-\left|\varphi_{n+1}\right|^{2}\right)-\frac{\partial H_{\mathrm{ph}}}{\partial u_{n}}
\end{aligned}
$$

The lowest energy states can be determined by numerical minimization of the functional $\mathcal{H}\left(\left\{\varphi_{n}\right\},\left\{u_{n}\right\}\right)=<\psi|\hat{H}| \psi>$. These solutions feature a stationary lattice. Other authors have computed DB solutions of the system as a whole, where both the lattice and the electron wavefunction vibrate with a common frequency - the solutions were dubbed "polarobreathers" [27,28]. However such solutions are most likely excited states. Instead, our starting point here are minimum energy states.

Figure 1 shows an example of a localized solution which is a minimum-energy state. It shows the time dependence of the real $\left\{\varphi_{n}^{r}\right\}$ and imaginary $\left\{\varphi_{n}^{i}\right\}$ part of the probability amplitudes $\left\{\varphi_{n}\right\}$, and of the displacements $\left\{u_{n}\right\}$. These and 
the other integrations of the equations of motion in this paper were performed with the RKSUITE routines [29]. This solution is a stationary solution analogous to that found in the Davydov model $[6,16,30]$ and known as the Davydov soliton. It includes a mixture of the soliton and DB characteristics, in that the lattice variables show a static, spatially localized distortion, characteristic of a soliton with zero velocity, while the quasiparticle is in a localized state with an internal vibration, and can be considered a stationary breather ${ }^{2}$. In keeping with our working definition of soliton and DB in the previous section, we suggest that such a state be called a solito-breather, or SDB, for short.

The internal frequency $\omega$ of the quasiparticle DB is related to the energy of the minimum energy state, as follows:

$$
\hbar \omega=<\psi_{0}\left|\left(\hat{H}_{\mathrm{qp}}+\hat{H}_{\mathrm{qp}-\mathrm{ph}}\right)\right| \psi_{0}>
$$

where $\mid \psi_{0}>$ is the SDB state determined by numerical minimization of the functional $\mathcal{H}$. Figure 2 shows the variation of this frequency with respect to the quasiparticle hopping parameter $V$ and the quasiparticle-lattice parameter $\chi$. There are two regimes, one in which the minimum energy states are spatially delocalized and another in which they are localized. As discussed elsewhere [31], the transition between the two is discontinuous. For delocalized states, the frequency $\omega=-2 V / \hbar$ varies linearly with $V$, as shown in the figure, while for localized, SDB states it varies nonlinearly. Depending on the relative values of $V, \chi, \kappa$ and $M$ it is possible to have cases in which the frequency $\omega$ is larger, of the same order, or smaller than that of the lattice vibrations. In the next section, states which arise under the action of different excitations of the SDBs, are explored for the three different cases.

\section{$3 \quad$ Excited states of solito-breathers}

Since the SDB in Fig. 1 has zero lattice momenta, one way of creating excitations varying the energy in a continuous manner is by adding non-zero momenta to that state. In this section the time evolution of such excitations is followed by using them as initial conditions in the equations of motion (7-8).

\footnotetext{
$\overline{2}$ This is of course a more restrictive application of the concept of breather, since the particular form of Eqs. (7-8) circumvents the problem of resonances, which is present in general systems. However, the solution still fits the description of DBs since it is the result of an interplay between discreteness and nonlinearity.
} 


\subsection{Low energy excitations}

Unless otherwise is specified, the excitations are obtained by adding to the minimum energy states, the SDB, momenta defined in the following manner:

$$
p_{n}=\lambda M\left(u_{n}-u_{n-1}\right)
$$

where $\lambda$ is real.

In Fig. 3, the system parameters are as in Fig. 1 and $\lambda \approx 0.03$. Although the lattice momenta oscillate, this has only a very small influence on the lattice distortion, which is not visible in the figure. However, the influence on the real and imaginary (not shown) parts of the probability amplitude is striking: on top of the oscillation associated with the initial SDB, there is modulation with a frequency 10 times smaller. The quasiparticle breather has thus become a multifrequency breather.

Larger values of $\lambda$, and thus greater initial momenta lead to different modulations for the quasiparticle wavefunction, having more than one frequency, as seen in Fig. 4 , for which $\lambda \approx 0.26$. A slight oscillation can now be seen in the lattice displacements. Increasing the strength of the initial momenta leads first to progressively larger amplitudes of the oscillation in the displacements. Initially, no radiation is lost since the induced lattice vibrations have a frequency out of resonance with the phonons of the extended system, Eq. 5. After a given size of the perturbation, the amplitude of these oscillations does not increase and instead the extra energy is deposited in radiation, as can be seen arising to the right of the central lattice distortion, in Fig. 5. Another remarkable fact is that the evolution of the momenta is the same, apart from the scale, in Figs. 3-5. In spite of very different magnitudes, the oscillations in the lattice momenta have roughly the same time dependence in all cases, and are only dependent on the initial values.

For other parameter values, in which the frequency of the quasiparticle oscillations are of the same order or slower than the lattice oscillations, low energy excitations do not have an influence on the quasiparticle oscillations. Higher energy excitations for the three cases are considered in the next subsection.

\subsection{Higher energy excitations}

In Fig. 5 the initial momenta are defined as $p_{n}=5.27526 M\left(u_{n}-u_{n-1}\right)$. This leads to localized oscillations in the momenta and localized oscillations in the lattice distortions, with a frequency that is 100 times smaller than that of 
the original SDB (compare timescale with Fig. 1). Figure 6 shows that, on a longer time scale, the excitation energy is split and two breathers arise: a central one, localized at the site of the initial SDB and a moving breather, with a lattice distortion approximately ten times smaller. The latter is just visible at 1.5 ps in Fig. 5, moving towards the end of the chain. In Fig. 6 it reaches the end of the chain at approximately $10 \mathrm{ps}$ and becomes visible coming out from the beginning of the chain (due to periodic boundary conditions). At approximately $20 \mathrm{ps}$ it hits the central breather and is scattered back to the beginning of the chain and, at the end of the simulation, it is then seen coming out of the end of the chain again. This moving breather can be seen both in the displacements and in the momenta. The amplitude of oscillation in the displacements associated with the moving breather is around zero, while that for the stationary breather is around the original stationary, localized profile of the minimum energy state.

In Fig. 7 the initial momenta are defined as $p_{n}=4.51844 M u_{n}$, so that although different from those used in Fig. 5 they correspond to trajectories in the same energy shell. Although the extra energy is the same as in Fig. 6, Fig. 7 shows that the amount of radiation generated in this case is much less. Indeed, in this case, all the excitation energy is deposited in the periodic oscillations of a stationary breather, located at the position of the lattice distortion of the minimum energy SDB state. The frequency of this stationary breather is approximately the same as the main frequency of the stationary breather in Fig. 6.

In Fig. 8, the system parameters were chosen so that the quasiparticle frequency $\omega$ is of the same order of magnitude as that of the oscillations in the lattice. These are generated by a high energy excitation with momenta defined in the same way as in Fig. 5. The immediate effect of the oscillations in the lattice variables on the quasiparticle is to create variations in the frequency of the quasiparticle breather, as seen in Fig. 8. Figure 9 shows that, in relative terms, more radiation is generated than in the simulations displayed in Figs. 5-7, but the stationary lattice breather remains localized even in the presence of such a large perturbation.

In Fig. 10, the system parameters were such that the quasiparticle frequency of the corresponding SDB was smaller than that of the lattice. A larger system was used, in order to delay the interference of the radiation which comes back to the centre of the chain because of the periodic boundary conditions. The timescale of the figures was also changed to fit the new frequencies of the lattice. Figure 10 shows that the perturbation creates two moving breathers (with opposite directions of propagation), together with a central stationary breather. The larger values of the lattice momenta are found in the moving breathers. Figure 11 shows that, although the moving breathers carry most of the kinetic energy of the lattice, they are not stable, and keep spreading. 
The central stationary breather, however, is stable and simulations with a spatially shorter system show that it survives the impact of the radiation in the unstable breathers. The quasiparticle frequency remains largely unaffected by the lattice dynamics.

In Fig. 12 an excitation of a broader SDB is considered. This again leads to full breather solutions, i.e. periodic oscillations both in the lattice and in the quantum quasiparticle. However, in this case, the central lattice breather moves. This is clearly seen in Fig. 12 where, approximately at 15 ps into the integration, the moving lattice breather is scattered by radiation coming from the boundaries and its direction is reversed. This is to be expected since, in general, the Peierls-Nabarro barrier that one has to overcome to move the polaronic structure is smaller as the structure becomes broader. However, although broader than the solutions considered in the previous figures, the solution displayed in Fig. 12 occupies six sites and can still be considered quite localized.

\section{Discussion}

In this article we study ILMs which arise as excitations of the minimum energy states of one quantum quasiparticle interacting with the vibrations of a nonlinear classical lattice. The minimum energy states of the system have been called solito-breathers (SDBs) since they consist of a quasiparticle periodic oscillation coupled to a stationary lattice soliton. A similar SDB state has also been studied in [32], where an electron is coupled to a Fermi-Pasta-Ulam lattice. The lattice distortion in this latter case is a kink.

The SDBs in our paper have zero lattice momenta and the study was restricted to excitations which arise because of finite initial lattice momenta. Apart from a scale factor, the time evolution of these excitations depend on the shape of the distribution of the initial momenta but not on their magnitude. $A$ general finding of this work is that the excitations of the minimum energy $S D B$ states are full breathers, i.e. both the quasiparticle and the lattice variables show periodic oscillations. As the excitation energy increases, these breathers become multifrequency breathers.

Although the excitation energy can be varied continuously, the states generated fall into different regimes. At very low energy, excitations with a single frequency modulation of the quasiparticle breather are observed, together with periodic oscillations in the lattice momenta. It is striking that in spite of their low energy, such excitations can have a profound influence in the frequencies of the quasiparticle breathers. No amount of radiation is lost to lattice phonons, since the frequency induced by the perturbations turns out to be out 
of resonance with the spectrum of linear excitations of the tails of the lattice, Eq. 5.

For higher energy excitations, periodic oscillations in the lattice displacements become clearly visible, and the quasiparticle breathers become multifrequency breathers. The amplitude of the lattice oscillations first increases with increasing excitation energy, until it reaches a saturation point. Higher excitation energies beyond this point lead to the generation of radiation. Part of this radiation is made up of moving breathers. Eventually, for sufficiently high excitation energies, e.g. corresponding to $\lambda \approx 11.5$, the quantum quasiparticle becomes less localized and when the lattice is populated by several moving breathers, it disperses (results not shown).

It was also found that the excitation of less localized SDBs can lead to moving breathers, in which a quasiparticle breather travels with an associated lattice breather. Such moving breathers are easily scattered by radiation or by other breathers.

The conclusion is that the excited states of the stationary (minimum energy) SBDs obtained by vibrational excitation are breathing states which keep localized with a remarkable robustness. This was found even in the presence of strong lattice radiation (although it is possible in this case that the solutions decay on a much longer time scale than the one studied here).

\section{Acknowledgements}

JLM acknowledges a Marie Curie TMR fellowship from the EU (No ERBFMBICT972761). JCE is grateful for EC support from RTN proposal no. RTN11999-00370.

\section{References}

[1] U. T. Schwarz, L. Q. English, and A. J. Sievers. Experimental generation and observation of intrinsic localized spin wave modes in an antiferromagnet. Phys. Rev. Lett., 83:223-226, 1999.

[2] B. I. Swanson, J. A. Brozik, S. P. Love, G. F. Strouse, A. P. Shreve, A. R. Bishop, W.-Z. Wang, and M. I. Salkola. Observation of intrinsically localized modes in a discrete low-dimensional material. Phys. Rev. Lett., 82(16):32883291, 1999.

[3] E. Trías, J. J. Mazo, and T. P. Orlando. Discrete breathers in nonlinear lattices: 
Experimental detection in a Josephson array. Phys. Rev. Lett., 84:741-744, 2000 .

[4] P. Binder, D. Abraimov, A. V. Ustinov, S. Flach, and Y. Zolotaryuk. Observation of breathers in Josephson ladders. Phys. Rev. Lett., 84:745-748, 2000 .

[5] G. Careri, U. Buontempo, F. Galluzzi, A. C. Scott, E. Gratton, and E. Shyamsunder. Spectroscopic evidence for Davydov-like solitons in acetanilide. Phys. Rev. B, 30:4689-4702, 1984.

[6] A. Scott. Davydov's soliton. Physics Reports, 217:1-67, 1992.

[7] J. C. Eilbeck, P. S. Lomdahl, and A. C. Scott. Soliton structure in crystalline acetanilide. Phys. Rev. B, 30:4703-4712, 1984.

[8] L. M. Floría, J. L. Marín, P. J. Martínez, F. Falo, and S. Aubry. Energy localisation in the dynamics of a Josephson junction ladder. Europhys. Lett., 36:539-544, 1996.

[9] A. J. Sievers and Shozo Takeno. Intrinsic localized modes in anharmonic crystals. Phys. Rev. Lett., 61(8):970-973, 1988.

[10] R. S. MacKay and S. Aubry. Proof of existence of breathers for time-reversible or Hamiltonian networks of weakly coupled oscillators. Nonlinearity, 7:16231643, 1994.

[11] S. Flach and C. R. Willis. Discrete breathers. Physics Reports, 295:181-264, 1998.

[12] S. Aubry. Breathers in nonlinear lattices: existence, linear stability and quantization. Physica D, 103:201-250, 1997.

[13] J. C. Eilbeck and R. Flesch. Calculation of families of solitary waves on discrete lattices. Phys. Lett. A, 149(4):200-202, 1990.

[14] D. B. Duncan, J. C. Eilbeck, H. Feddersen, and J.A.D. Wattis. Solitons on lattices. Physica D, 68:1-11, 1993.

[15] J. C. Eilbeck, P. S. Lomdahl, and A. C. Scott. The discrete self-trapping equation. Physica D, 16:318-338, 1985.

[16] A. S. Davydov. Solitons in Molecular Systems. D. Reidel, Dordrecht, Boston, Lancaster, Tokyo, 1985.

[17] T. Holstein. Studies of polaron motion. part I. the molecular crystal model. Ann. Phys., 8:325-342, 1959.

[18] T. Holstein. Studies of polaron motion. part II. the "small" polaron. Ann. Phys., 8:343-389, 1959.

[19] A. S. Davydov and A. V. Zolotariuk. Autolocalized collective excitations in molecular chains with cubic anharmonicity. Phys. Status Solidi B, 115:115125, 1983. 
[20] V. M. Kenkre. In S. Pnevmatikos, T. Bountis, and Sp. Pnevmatikos, editors, Singular behaviour and nonlinear dynamics, volume II, Singapore, 1989. World Scientific.

[21] H.-L. Wu and V. M. Kenkre. Multiple stationary states and saturation effects for rotational polarons. Phys. Lett. A, 199:61-64, 1995.

[22] V. M. Kenkre. What do polarons owe to their harmonic origins? Physica D, 113:233-241, 1998.

[23] S. Raghavan, A. R. Bishop, and V. M. Kenkre. Quantum versus semiclassical description of self-trapping: Anharmonic effects. Phys. Rev. B, 59:9929-9932, 1999.

[24] Y. Gaididei, N. Flytzanis, A. Neuper, and F. G. Mertens. Effect of nonlocal interactions on soliton dynamics in anharmonic lattices. Phys. Rev. Lett., 75:2240-2243, 1995.

[25] L. Cruzeiro-Hansson and S. Takeno. Davydov model: The quantum, mixed quantum-classical, and full classical systems. Phys. Rev. E, 56(1):894-906, 1997.

[26] L. Cruzeiro-Hansson and V.M. Kenkre. Comparison of quantum Monte Carlo and semiclassical Monte Carlo results in investigations of thermal stability of Davydov solitons. Phys. Lett. A, 203:362-366, 1995.

[27] G. Kalosakas and S. Aubry. Polarobreathers in a generalized holstein model. Physica D, 113:228-232, 1998.

[28] G. Kalosakas, S. Aubry, and G.P. Tsironis. Polaron solutions and normal mode analysis in the semiclassical holstein model. Phys. Rev. B, 58:3094-3104, 1998.

[29] R. W. Brankin, I. Gladwell, and L. F. Shampine. RKSUITE: a suite of Runge-Kutta codes for the initial value problem for ODEs. Softreport 92S1, Department of Mathematics, Southern Methodist University, Dallas, Texas, U.S.A, 1992.

[30] L. Cruzeiro-Hansson and V.M. Kenkre. Localized versus delocalized ground states of the semiclassical Holstein Hamiltonian. Phys. Lett. A, 190:59-64, 1994.

[31] L. Cruzeiro-Hansson, J. C. Eilbeck, J. L. Marín, and F. M. Russell. Interplay between dispersive and non-dispersive modes in the polaron problem. Phys. Lett. A, 266:160-166, 2000.

[32] Y. Zolotaryuk and J. C. Eilbeck. A breather-like localised mode in a FPU lattice interacting with an electron. J. Phys.: Condens. Matter, 10:4553-4564, 1998. 

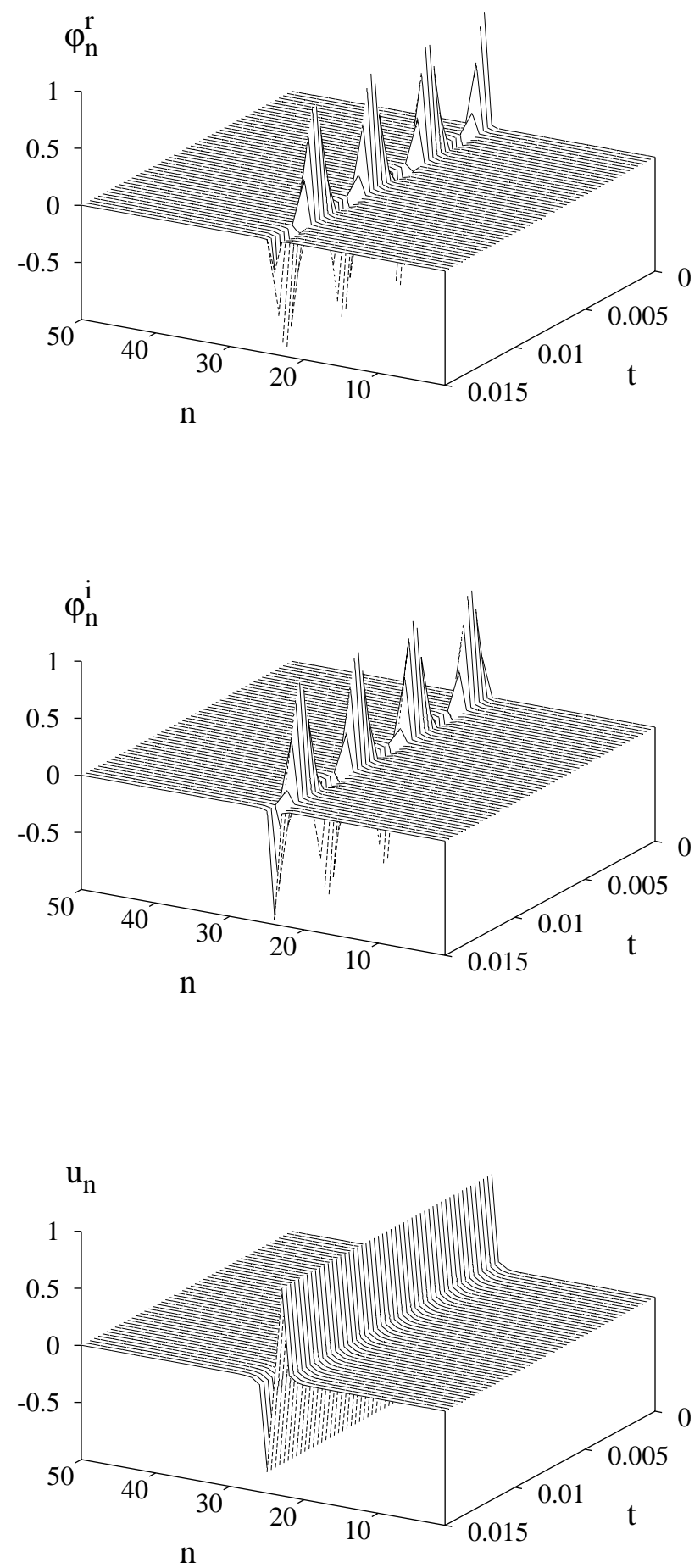

Fig. 1. Time dependence in picoseconds of the real $\varphi_{n}^{r}$ and imaginary $\varphi_{n}^{i}$ parts of the probability amplitude for a quantum quasiparticle in site $n$, and of the lattice displacement of site $n, u_{n}$, in $\AA$. The system parameters were: $V=30 \cdot 10^{-22} \mathrm{~J}$, $\chi=1000 \mathrm{pN}, \kappa=1 \mathrm{~N} / \mathrm{m}, \kappa^{\prime}=2 \kappa$ and $M=0.95 \cdot 10^{-23} \mathrm{Kg}$. 


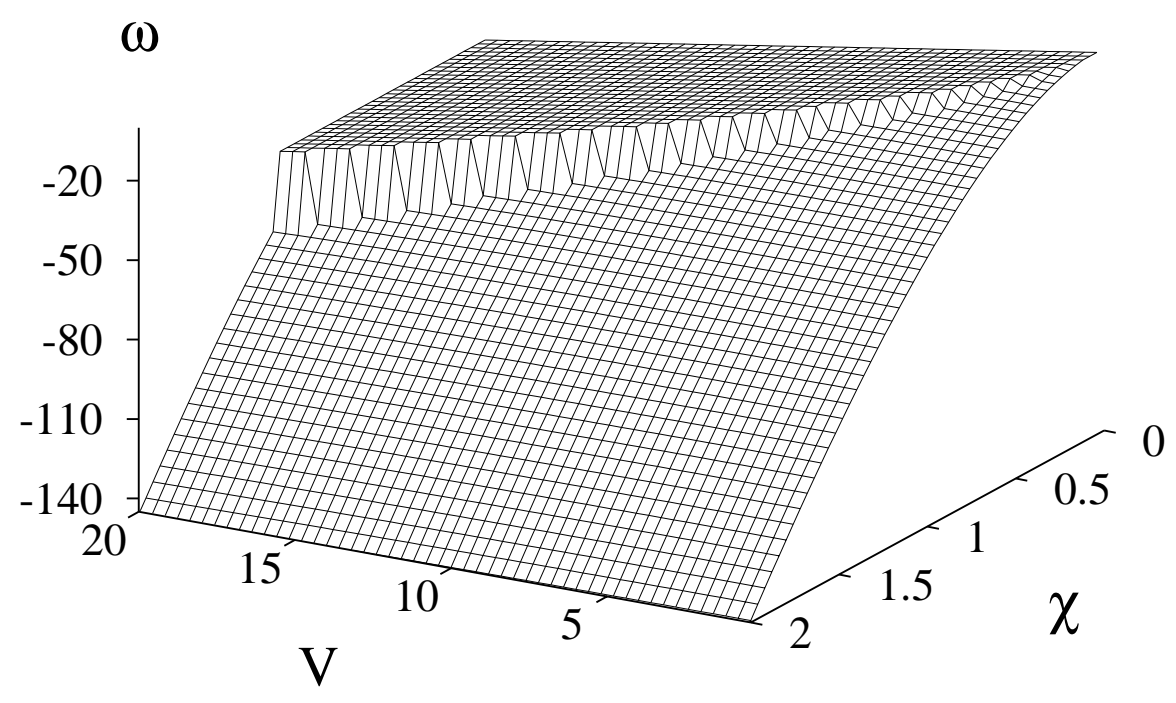

Fig. 2. Variation of the quasiparticle breather frequency $\omega$ with the quasiparticle hopping strength $V$ and the quasiparticle-lattice coupling parameter $\chi$. 

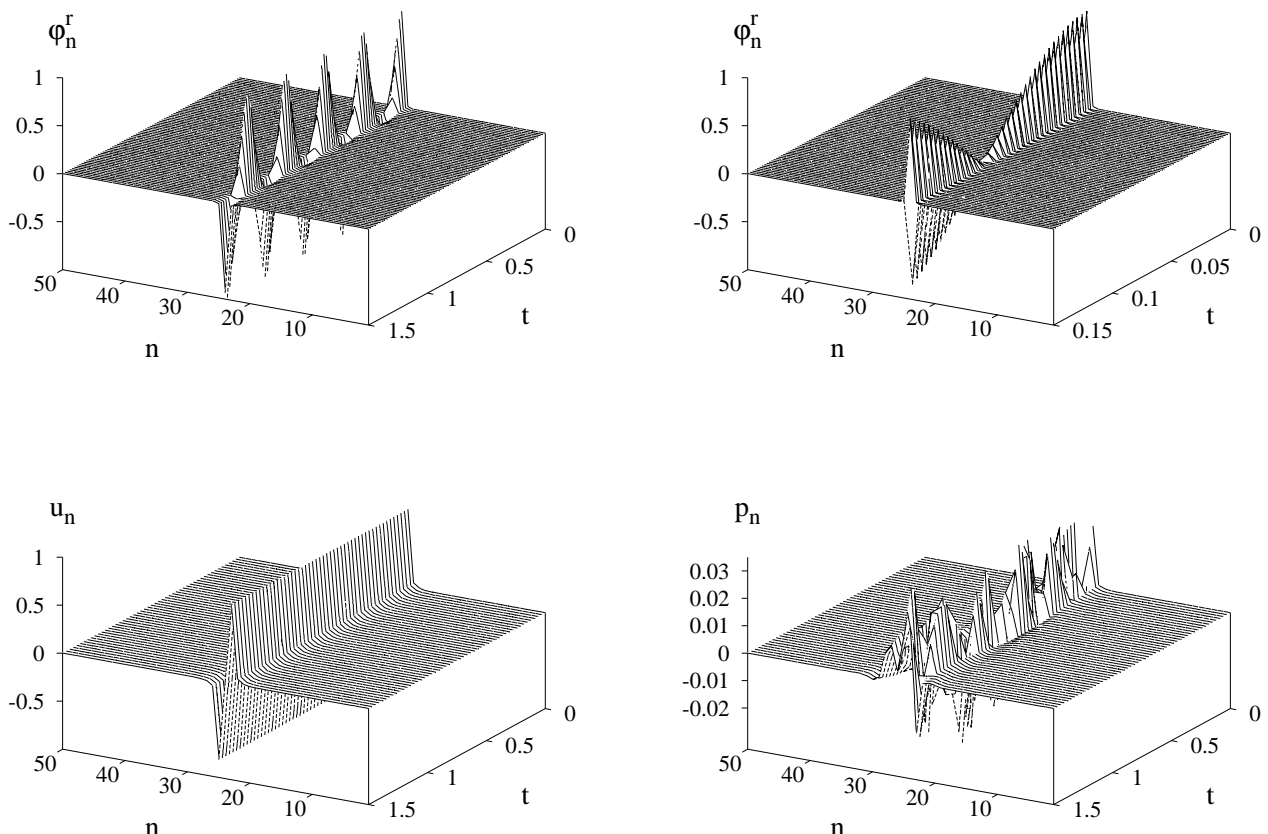

Fig. 3. Time dependence, in picoseconds, of the real part of the probability amplitude for a quantum quasiparticle in site $n, \varphi_{n}^{r}$, and of the lattice displacement, $u_{n}$, in $\AA$, and momenta, $p_{n}$, in $M \cdot \AA / \mathrm{ps}$, of site $n$. The system parameters are as in Fig. 1 . The initial momenta are defined as $p_{n}(0)=0.02968 M\left(u_{n}-u_{n-1}\right)$. 

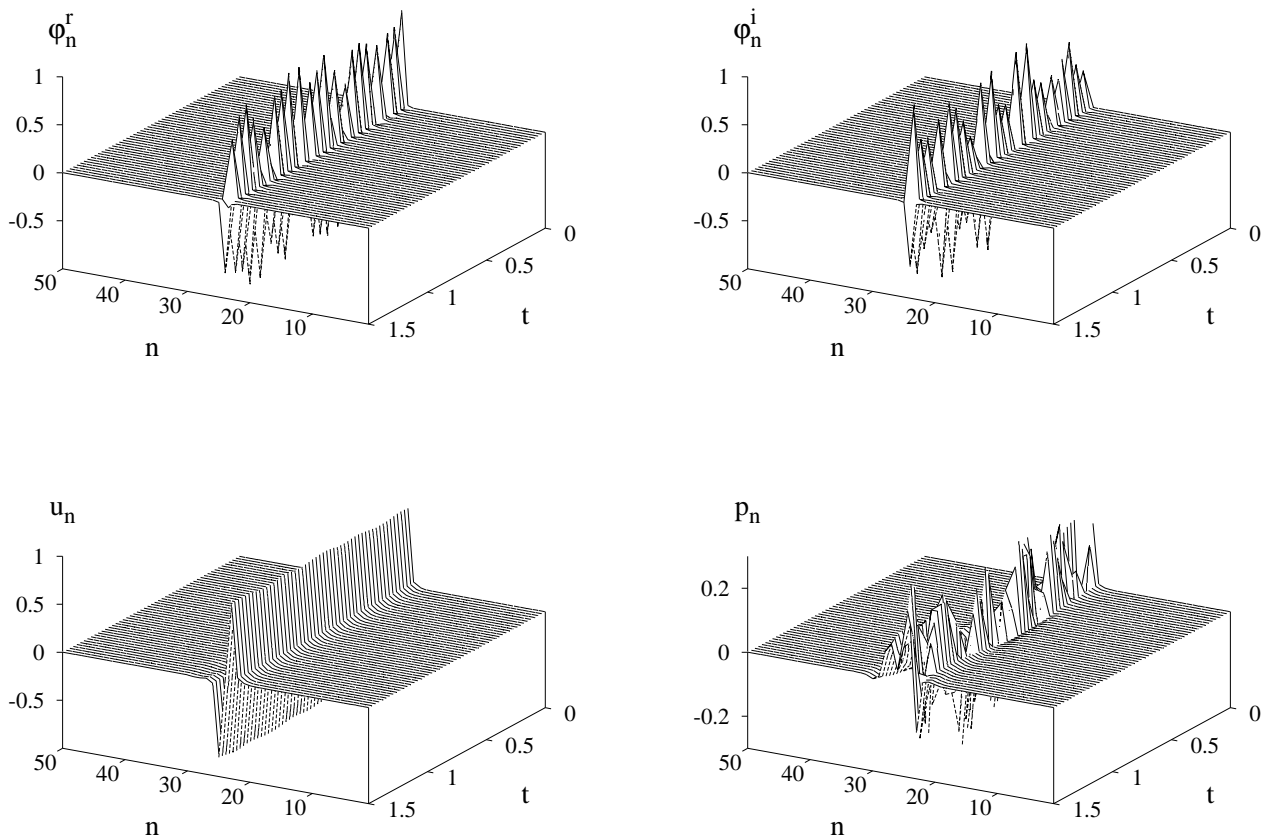

Fig. 4. Same as in Fig. 3, but where the initial momenta are defined as $p_{n}(0)=0.26111 M\left(u_{n}-u_{n-1}\right)$. 

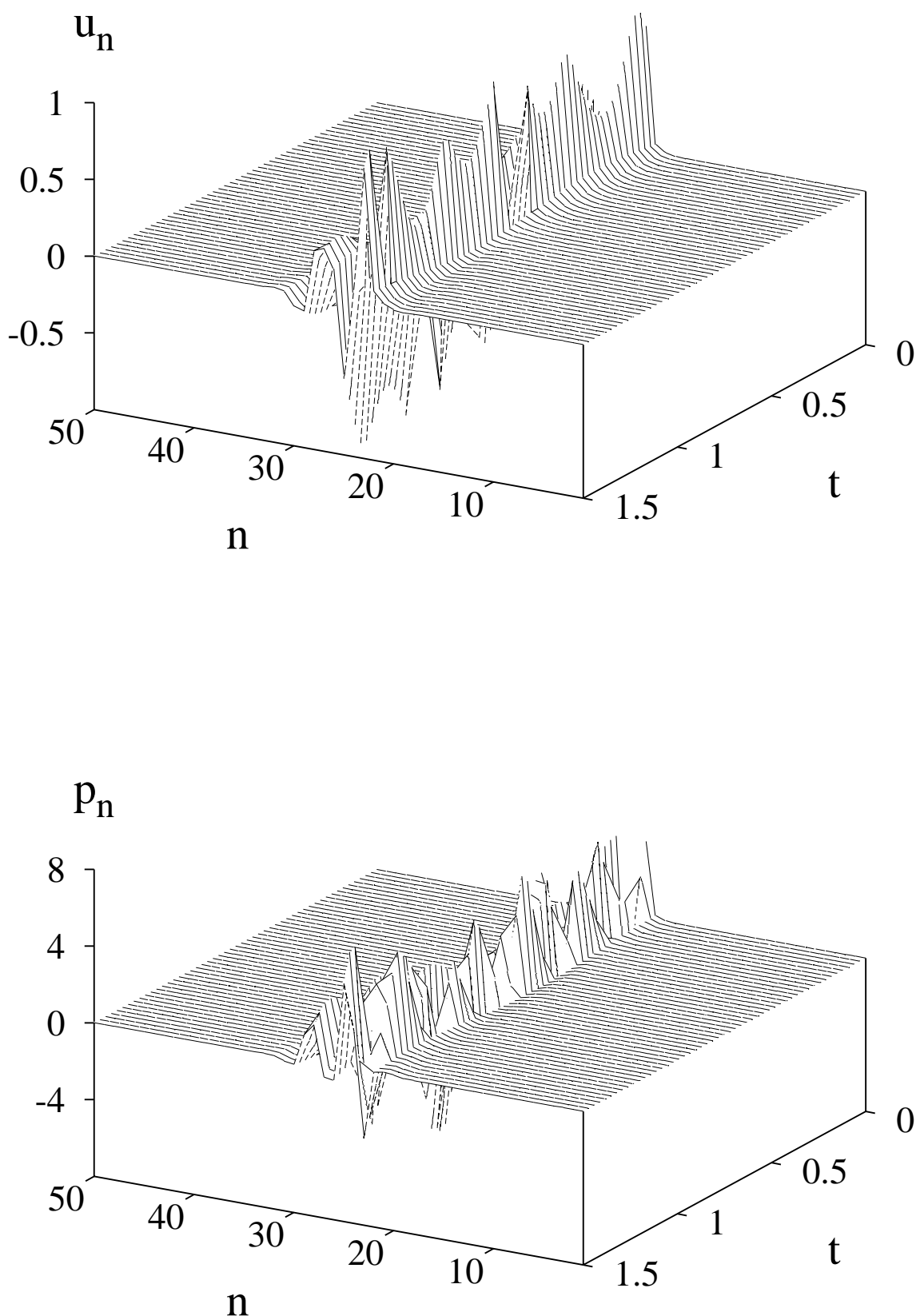

Fig. 5. Time dependence, in picoseconds, of the lattice displacement, $u_{n}$, in $\AA$, and momenta, $p_{n}$, in $M \cdot \AA / p s$, of site $n$. The system parameters are as in Fig. 1 . The initial momenta are defined as $p_{n}(0)=5.27526 M\left(u_{n}-u_{n-1}\right)$. 

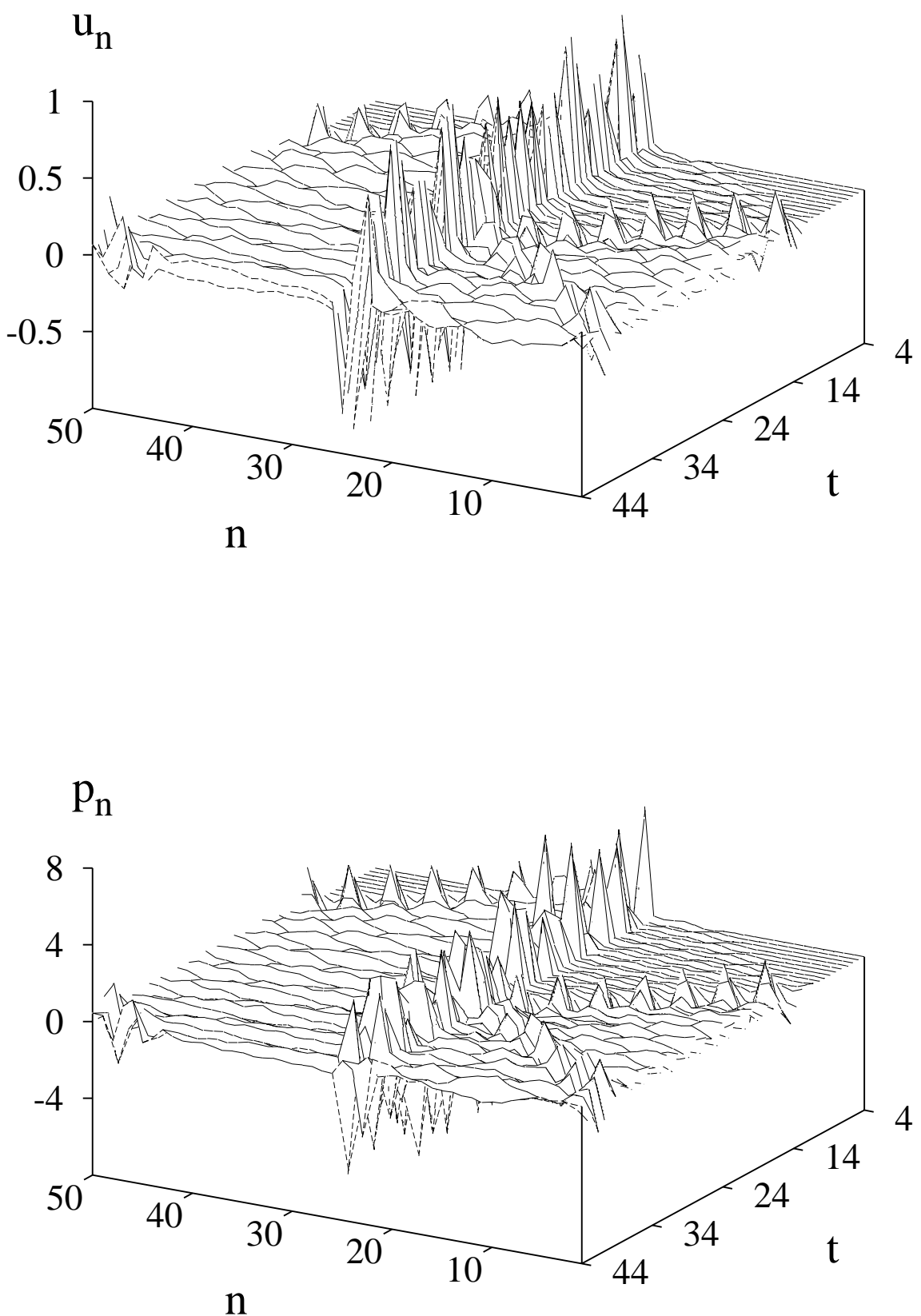

Fig. 6. Same as Fig. 5, for a longer time. 

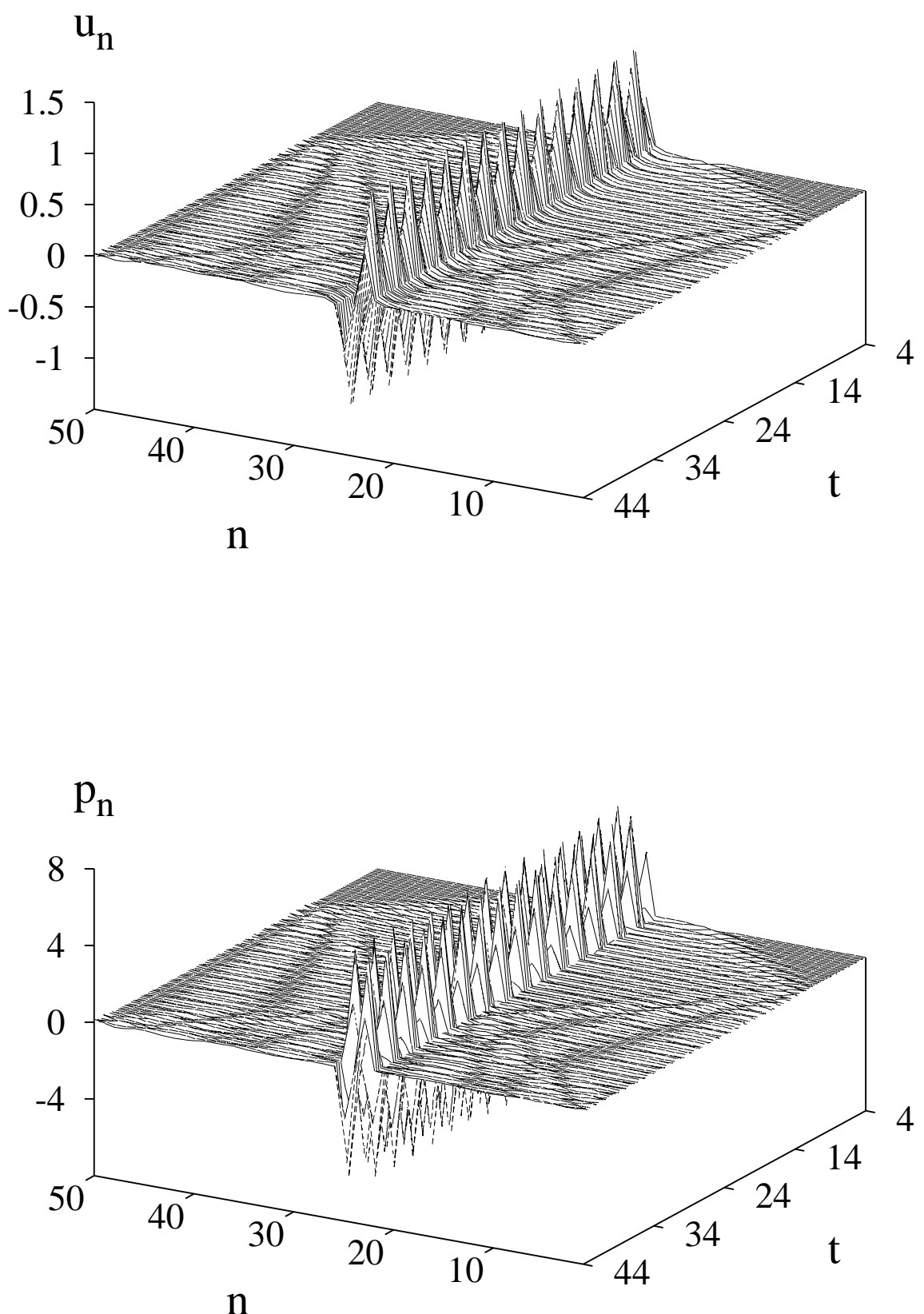

Fig. 7. Same as Fig. 5, but with initial momenta defined as $p_{n}(0)=4.51844 M u_{n}$, so that energy is the same as in Figs. 5, 6. 

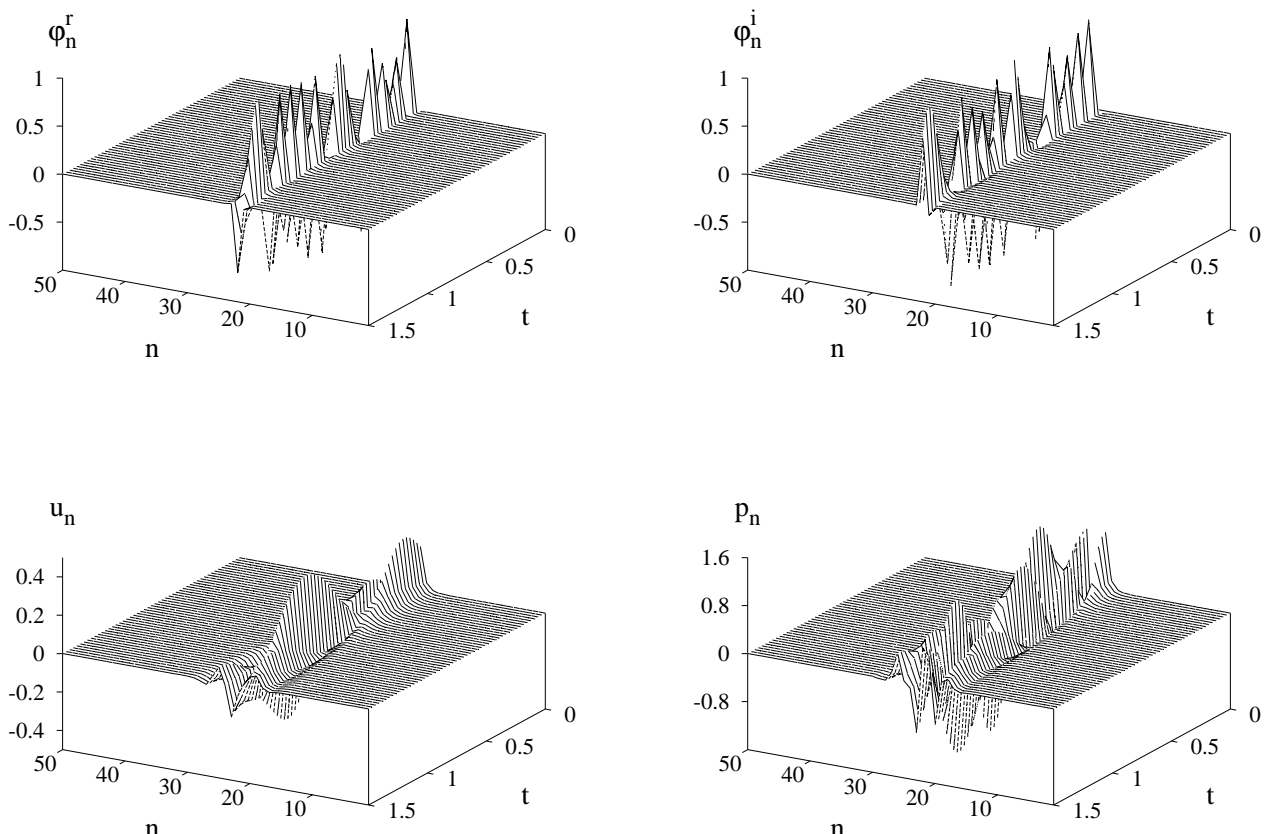

Fig. 8. Same as in Fig. 5, but with new system parameters, as follows: $V=0.3 \cdot 10^{-22}$ $\mathrm{J}$ and $\chi=100 \mathrm{pN}$.

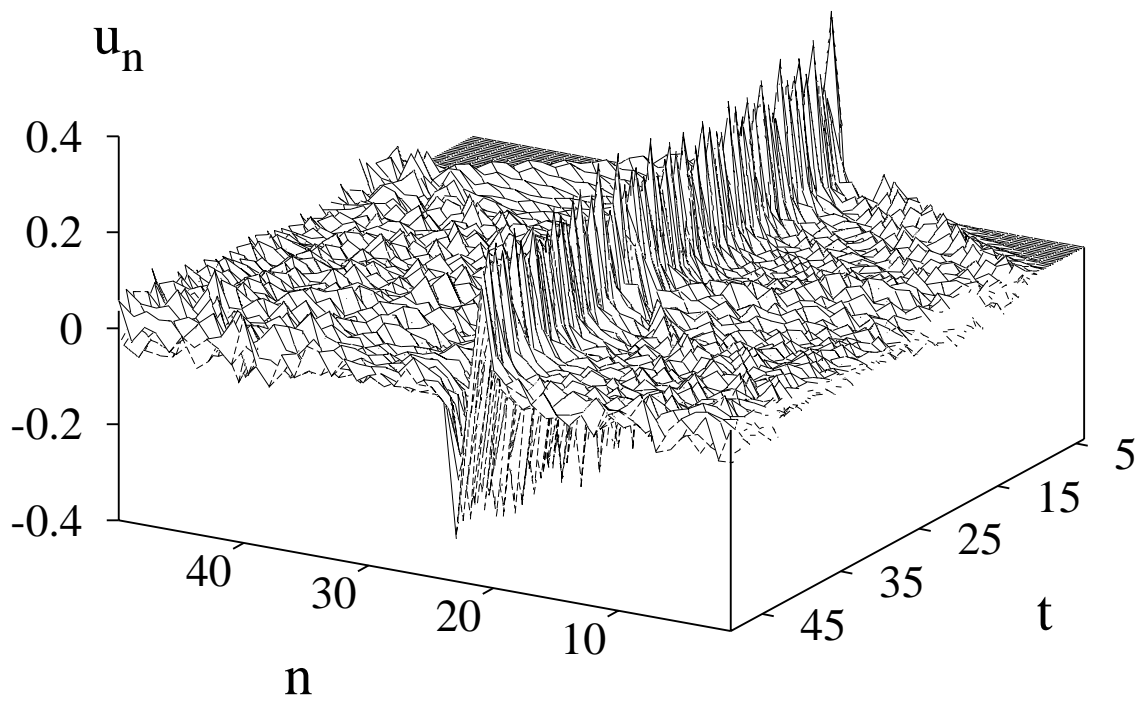

Fig. 9. Continuation of time dependence of displacements in Fig. 8. 

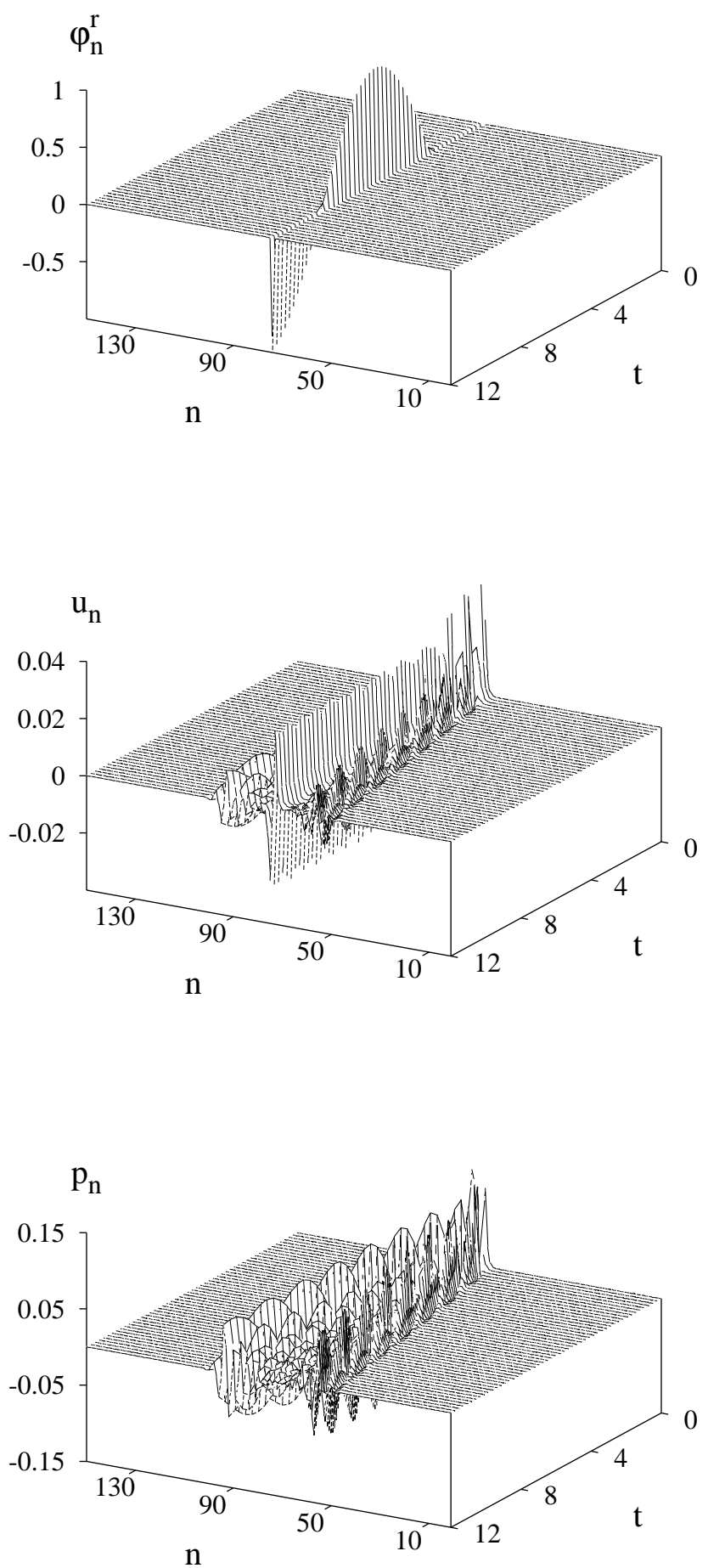

Fig. 10. Same as in Fig. 5, but with new system parameters, as follows: $V=0.003 \cdot 10^{-22} \mathrm{~J}$ and $\chi=10 \mathrm{pN}$. 

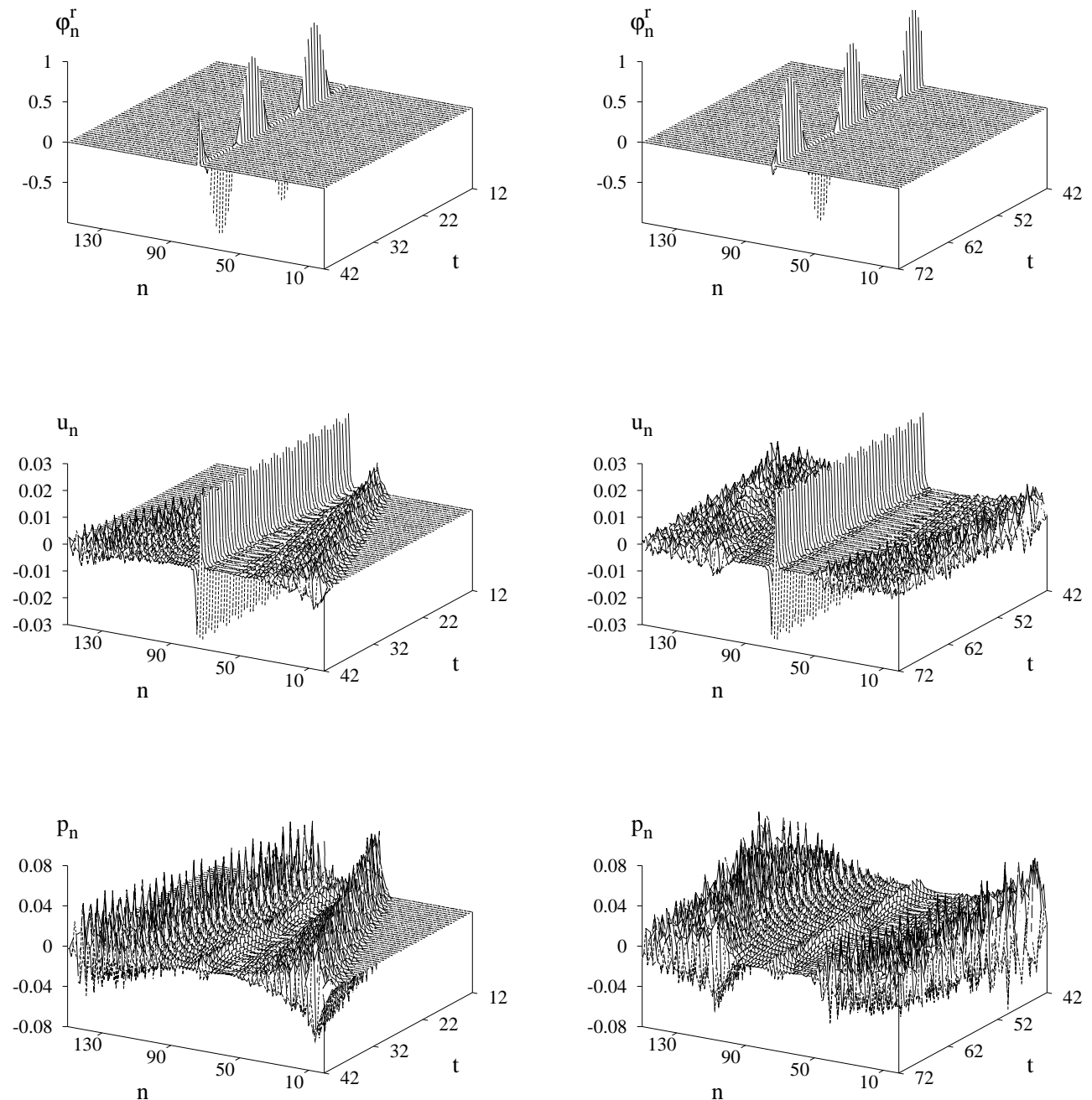

Fig. 11. Same as Fig. 10, at a later time. 

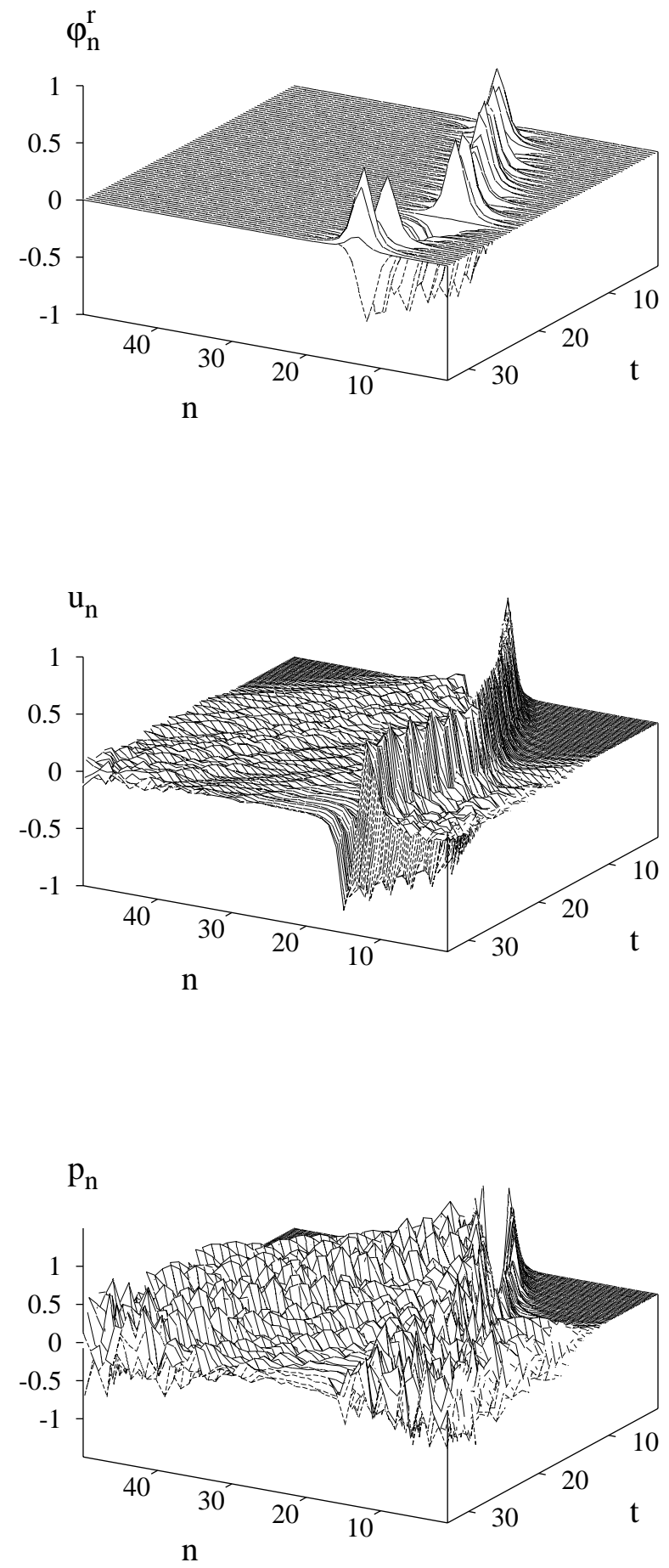

Fig. 12. Same as in Fig. 1, but $V=1000 \cdot 10^{-22} \mathrm{~J}$ and initial momenta are defined as $p_{n}(0)=2.07818 M\left(u_{n}-u_{n-1}\right)$. 